\title{
Application of Biomarkers and Biosensors to Detect and Track Pathogenic Agents
}

\author{
Hamidreza Shirzadfar* and Mahtab Khanahmadi \\ Department of Biomedical Engineering, Sheikhbahaee University, Esfahan, Iran
}

Received:06 June, 2018; Accepted: 25 June, 2018; Published: 26 June, 2018

*Corresponding author: Hamidreza Shirzadfar, Department of Biomedical Engineering, Sheikhbahaee University, Esfahan, Iran; E-mail: h.shirzadfar@ shbu.ac.ir

\begin{abstract}
Detection and identification of pathogenic bacteria and cancer cells is important because it is one of the most important factors in mortality among different societies. There are many laboratory methods to identify bacteria that are very time consuming. A long time in diagnosing a bacterium causes the bacteria to grow more and the patient gets worse. Biomarkers and biosensors can identify pathogenic agents and detect them. By using these methods, bacteria and diseases can be detected more quickly and more safely. Using biomarkers, drugs can be delivered to the target tissue so that other tissues are not damaged.
\end{abstract}

\section{Introduction}

Biomarkers are an indicator for measuring and detecting pathogenic factors and assessing biological conditions. Biomarkers in the field of cancer diagnosis and treatment have been widely considered and have been widely used in these fields. Biomarker is an indicator of a specific disease and physical states in the living tissue, and the biomarker can detect the symptoms of a specific disease. Some biomarkers are delivered the drug to the tissue that the biomarker is labeled with that drug and is transmitted to the target tissue. Markers are divided into molecular and phenotypic types, and molecular types are available in both biochemical and DNA $[1,2]$.
A biosensor is a biological device that is used in various fields, such as rapid detection and traceability of pathogens, blood glucose measurements, contamination control, DNA analysis, and the study of the effects of drugs. Biosensors have Bioreceptor and Transducers. Bioreceptor has been created from biological molecules, which analytes are placed on this part and then the Physicochemical Changes are converted into electrical signals. These signals are converted to voltage values, and based on these values, the amount of Biomarkers and biosensors should be biocompatible and not dangerous to the tissues and cells of the body and should also be Immobilized and have no negative effect on other tissues in the body. Biomarkers and biosensors should be chemically stable in living tissue $[4,5]$.

\section{Method}

Biologic material can be detected. Bioreceptor can be from DNA, enzyme, antibody, and so on. Transducers used in biosensors are electrochemical, piezoelectric, optical and thermal, which are used in various fields [Figure 1]. Using biomarkers and biosensors, bacteria and pathogenic agents can be detected and traced, and biomarkers and biosensors can be used to deliver the drug to the target tissue.

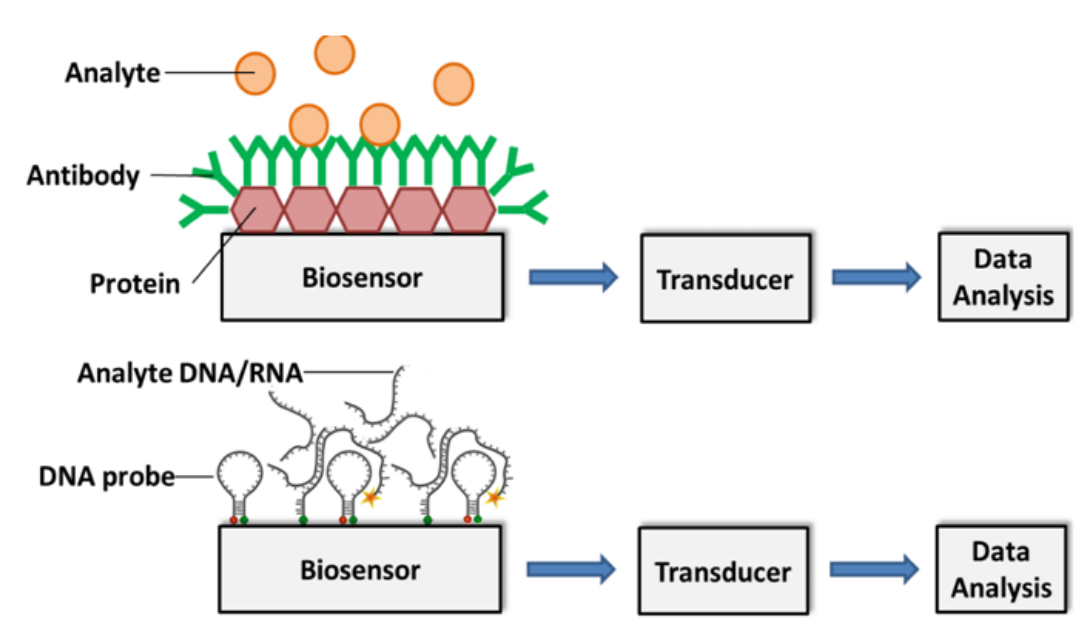

Figure 1: Biosensor function [3] 


\section{Biomarkers}

Detection of Escherichia coli (E. coli) in water: Escherichia coli are a gram negative bacilli species of Enterobacteriaceae family that is present in the lower intestine of warm-blooded animals. This bacterium leads to food poisoning and diarrhea in humans. To detect this bacterium, a strip of whatman paper containing glucose is used to absorb the bacterium. Other parts of the bar are hydrophobic, which prevent the cells from stopping. A bacterium that comes to the paper is immersed in the strip based on by using the detector biosensors, E. coli can be identified, which is based on specific molecules present on the surface of the bacterium. Also, using biosensors, it can be detected by calorimetry method, and through DNA, this bacterium in food [9]. The capillary property and reaches the reactive region. This reactive region has a growth environment for galactose that decomposes by Escherichia coli. This method has a positive result for $200 \mathrm{CFU} / \mathrm{ml}$ Escherichia coli, but similar methods can have the correct result for a smaller number of Escherichia coli cells in the sample [6,7] [Figure 2].

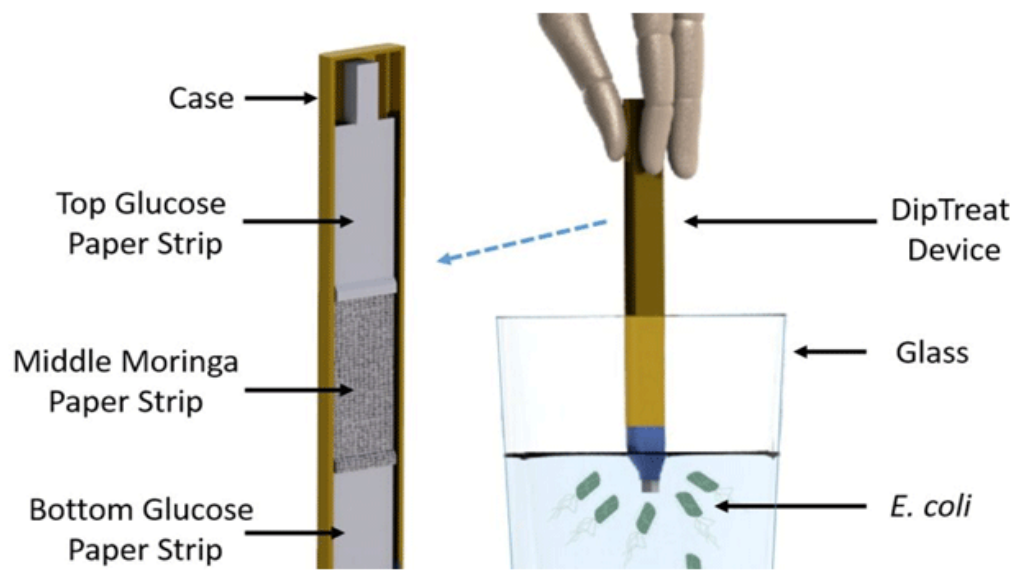

Figure 2: Detection of E. coli in water with whatman paper [8]

Detection of Sepsis Disease: In sepsis, microbial pathogens attack the bloodstream and the immune system, causing infection in the blood. There are about 150 biomarkers to diagnose the disease, which are structurally included: cell markers, coagulation markers, endothelial vascular biomarkers, cytokine markers, and Acute-Phase proteins [10, 11]. The sCD14-ST is a new marker for the diagnosis of the disease, which has been very much considered. CD14 is a glycoprotein that is on the surface of monocytes. CD14 is a receptor for lipopolysaccharides or lipopolysaccharides proteins [12] [Figure 3].

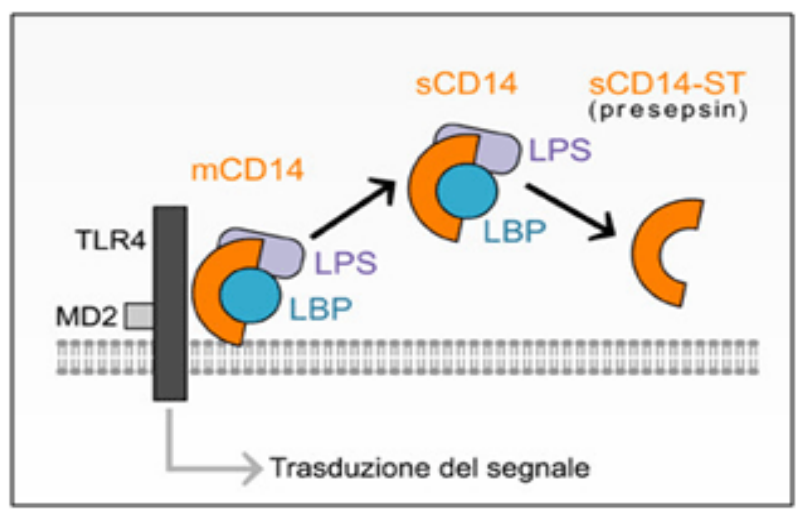

Figure 3: The sCD14-ST Biomarker
mCD14: membrane CD14; sCD14: soluble CD14; sCD14ST: soluble CD14 Subtype; LPS: Lipopolysaccharides; LBP: Lipopolysaccharide Binding Protein; TLR4: Toll-like Receptor 4; MD2: TLR4 Co- Protein [13].

C - reactive protein (CRP), which is part of the Acute-Phase protein, is also a non- Glycosylation protein. This protein is made by the liver and released in the blood when it is infected. By increasing this protein in the blood, can be detection infection and inflammation. This method has been used for many years [14] [Figure 4].

Heparin binding proteins are released from neutrophilic secreted vesicles and have antimicrobial activity. Sepsis can be diagnosed by counting the levels of these proteins along with white blood cell count. This marker is a valuable and validated diagnostic method for diagnosing sepsis [16]. Detection of Pneumonia Disease: Pneumonia is a respiratory infection in which the lungs become inflamed. In this disease, Alveoli are damaged and the disease is caused by bacteria and viruses. Plasma Hepcidin, a peptide hormone as a biomarker, can detect pneumonia. Based on the blood sample taken, Plasma Hepcidin levels in this type of disease are significantly increased $[17,18]$. Detection of Myocardial Infarction: Myocardial infarction causes a heart attack that causes coronary artery occlusion and leads to cell death in a part of the heart muscle. The cardiac troponin marker can be used 12 hours after the onset of the disease, which is a long time for 12 hours and may be worsening over time [19, 20]. The heart fatty acid binding protein (H-FABP) is a rapid 


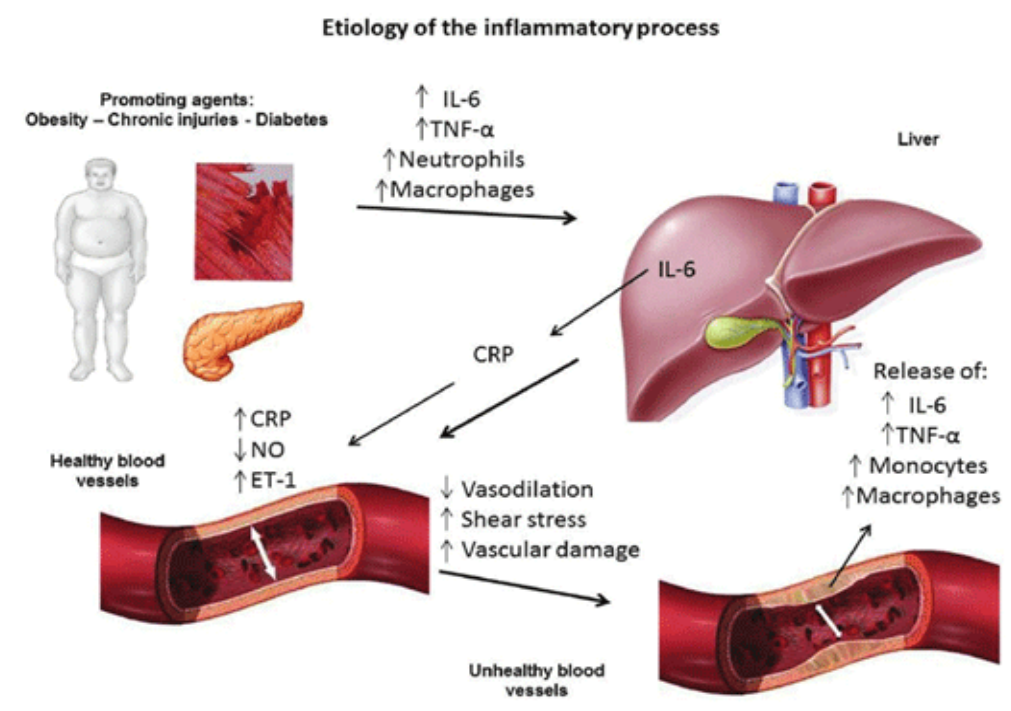

Figure 4: Detection inflammation with C - reactive protein Biomarker, IL-6: Interleukin 6; TNF- $\alpha$ : Tumor Necrosis factor alpha; EN-1: Endothelin-1; NO: Nitric Oxide [15]

marker that can detect heart attacks in about one to five hours. Also, with this biomarker, can be quickly diagnose inflammatory myocarditis. This marker is available at high concentrations in myocytes [21]. Cancer cells can be detected using micro-RNA (miRNA). The highest amount (miRNA) in the circulation, along with lipid and lipoprotein. miR-17-5p, miR-21, miR-106a are diagnostic biomarkers for gastric cancer [22].

\section{Biosensors}

Aptamers are single-stranded oligonucleotide ligands that can be rotated. Prostate cancer can be detected using electrochemical biosensors based on Aptamers. Also, these types of sensors are

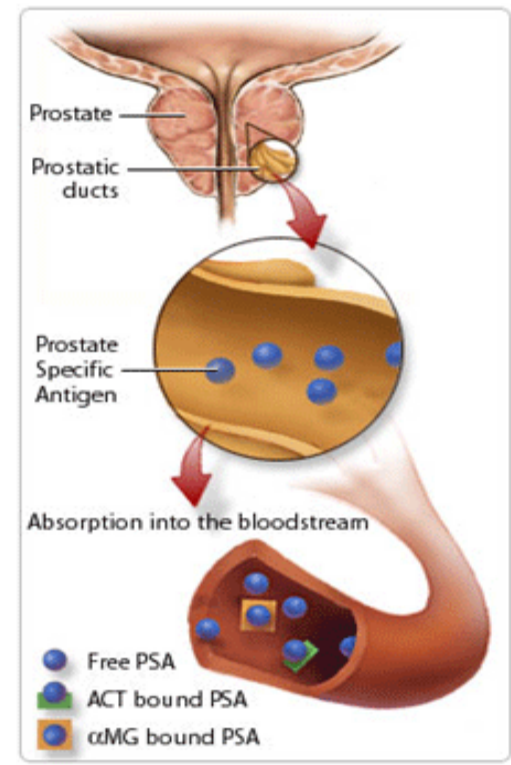

Figure 5: Detection prostate cancer with PSA, ACT: Anti-chymotrypsin; $\alpha M G$ : alpha-Macroglobulin [27] called Aptasensor. Electrochemical Aptasensor act on the basis of electrochemical current changes, which are after the interaction of the target molecule with Aptamer $[23,24]$. The best biomarker for diagnosing prostate cancer is a Prostatic Specific Antigen (PSA), which is a serine protease and is made up of glucose and peptide. PSA is secreted by healthy and cancerous cells of the prostate, which increases when it occurs during cancer. With electrochemical Aptasensor, can be measure the amount of PSA and monitor and measure prostate cancer [25, 26] [Figure 5].

There are various methods to measure glucose using biosensors. Blood glucose levels can be detected in urine using biosensors made of copper oxide (Cu2O). Also, with biosensors along with Indium (III) oxide (In203), Chitosan and glucose oxidase enzymes, glucose levels can be determined in saliva, tears and sweat $[28,29]$. Bioluminescence Microbial Biosensor is a fast, precise, and sensitive tool used to measure and detect contamination and environmental bacteria in micrometer scale [30-31]. By using a nanobiosensor, Salmonella typhi can be recognized as a causative agent of typhoid fever. This method is based on the response between the antigen and the antibody which is based on fluorescence. Biosensors that contain fluorescence particles of gold and silicon, bind to Salmonella bacteria that are in food, and detect this bacterium [32, 33]. A series of biosensors are very sensitive to the gas created from food corruption, which, as a result, changes their color. Using these sensors, these pathogens can be detected in food [34]. The Yersinia Pestis bacteria that causes plague disease can be detected using fiber optic biosensors, which has high speed and precision [35]. Biosensors can identify pathogenic factors in the soil and recognize the useful and harmful soil organisms. The function of this method is based on the amount of oxygen consumed [36]. Biosensors are also used to detect mercury levels in aquatic animals. In this method, it is possible to detect the amount of mercury and bacteria from this mercury by irradiation 
[37].Using the biosensors made of gold nanowire, bacteria in the kidney infection can be detected [38]. Biosensors can examine the quality of cereals and detect pathogens in cereals with polymer nanoparticles. Also, acoustic biosensors can quickly detect pathogens in the water [39].

\section{Advantages and Disadvantages}

Biosensors and biomarkers, due to the fact that they are composed of biological substances, do not have side effects and harmful effects on the living tissue and do not cause toxicity in the body [40]. Biosensors can quickly and continuously control and measure metabolic and biological activity. Biosensors can measure non-polar compounds that are produced in living tissue. Biomarkers and biosensors are proprietary and designed for the intended purpose and therefore very well function. Also, the biosensors are very fast in the analysis, and they perform a quick and direct analysis. Bioreceptor and transducers are placed in a sensor, and the detection and conversion of the sensor is carried out simply and quickly, and does not require multiple steps. Biosensors, which are biological elements, can be re-produced and re-used, and not disposable, which can also be traced continuously. Therefore, biosensors are a powerful, precise, safe and fast tool for tracking and detecting bacteria and biological molecules. Biomarkers have a lot of efficiency and effectiveness in frozen samples experiments. Also, these biomarkers act in a special way to study the blood mass and do not react with other blood factors. In some cases, biomarkers can predict the disease before it starts. Biomarkers are detectable and traceable in all tissues. Some of the enzymes used in the biosensors are unsuitable stability and require cofactors for stability and catalytic activity. In some cases, marking biosensors and biomarkers is difficult and complex and requires complex reactions and costs [41-42].

\section{Conclusion}

Biomarkers and biosensors, due to the fact that they are made of biological materials, are harmless to the tissues of the body, and can also detect bacteria and pathogenic agents more quickly and accurately. By using these methods, drugs can be delivered accurately and quickly to target tissues. Biomarkers and biosensors can quickly and accurately detect diseases in some cases before they begin. With this method, the treatment of diseases is greatly improved. Biomarkers and biosensors can identify specific and dangerous bacteria in food that prevent poisoning and side effects, and reduce mortality from bacteria.

\section{References}

1. Hayes J, Peruzzi PP, Lawler S MicroRNAs in cancer: biomarkers, functions and therapy. Trends Mol Med. 2014; 20(8):460-469. Doi: 10.1016/j.molmed.2014.06.005

2. Kurt AJ, Gregor KW. Multiple system atrophy: pathogenic mechanisms and biomarkers. Journal of Neural Transmission. 2016;123(6):555572

3. Yi Fang, Ramaraja PR. Current and Prospective Methods for Plant Disease Detection. Biosensors. 2015; 5(3):537-561. Doi:10.3390/ bios5030537

4. Shirzadfar H. Design and evaluation of a GMR-biosensor for magnetic characterization of biological medium. IJL - Institut Jean Lamour. 2014;1-157.

5. A Taheri-Kafrani, H Shirzadfar, E Tavassoli-Kafrani. Dendrimers and Dendrimers-Grafted Superparamagnetic Iron Oxide Nanoparticles: Synthesis, Characterization, Functionalization, and Biological Applications in Drug Delivery Systems. Nano- and Microscale Drug Delivery Systems. 2017;75-94. Doi: 10.1016/B978-0-323-527279.00005-4

6. Shih CM, Chang CL, Hsu MY, Lin JY, Kuan CM, Wang HK, et all., Paperbased ELISA to rapidly detect Escherichia coli. Talanta. 2015;145(1):25. Doi: 10.1016/j.talanta.2015.07.051

7. Stauber C, Miller C, Cantrell, B Kroell K. Evaluation of the compartment bag test for the detection of Escherichia coli in water. Journal of Microbiological Methods. 2014;99:66-70. Doi: 10.1016/j. mimet.2014.02.008

8. https://www.glaciercleantech.com/wp-content/uploads/2017/03/ dipTreat-900x500.jpg

9. Shirzadfar H,Nadi M, Kourtiche D, Yamada S, Shahabi P. Characterization Of A Needle-Type Giant Magnetoresistance Sensor For Detection Of Escherichia Coli's Magnetic Marker. 2015;8(1):220-234.

10. Prucha M, Bellingan G, Zazula R. Sepsis biomarkers. Clin Chim Acta. 2015;440:97-103. Doi: 10.1016/j.cca.2014.11.012

11. Xiao Liu, Hui Ren, Daizhi Peng. Sepsis biomarkers: an omics perspective. Frontiers of Medicine. 2014;8(1):58-67.

12. Chenevier-Gobeaux C, Borderie D, Weiss $\mathrm{N}$, Mallet-Coste $\mathrm{T}$ Claessens YE. Presepsin (sCD14-ST), an innate immune response marker in sepsis. Clin Chim Acta. 2015;450:97-103. Doi: 10.1016/j. cca.2015.06.026

13.http://www.gepasrl.it/images/presepsin_r1_c1.jpg

14. Waheeda Nargis, Ibrahim MD, Borhan Uddin Ahamed. Procalcitonin versus C-reactive protein: Usefulness as biomarker of sepsis in ICU patient. Int J Crit Illn Inj Sci. 2014;4(3):195-199. Doi: 10.4103/22295151.141356

15. http://www.beautiful-elegance.com/wp-content/uploads/2018/03/ inflammatory-markers-c-reactive-protein.jpg

16. Kjolvmark CH, Pahlman L, Akesson P, Linder A. Heparin-Binding Protein: A Diagnostic Biomarker of Urinary Tract Infection in Adults. Open Forum Infect Dis. 2014;1(1):1-9. Doi: 10.1093/ofid/ofu004

17.Wang $\mathrm{CH}$, Babitt $\mathrm{JL}$. Hepcidin regulation in the anemia of inflammation. Curr Opin Hematol. 2016;23(3):189-197. Doi: 10.1097/ MOH.0000000000000236

18. Michels KR, Zhang Z, Bettina AM, Cagnina RE, Stefanova D, Burdick $\mathrm{MD}$, et al. Hepcidin-mediated iron sequestration protects against bacterial dissemination during pneumonia. JCI Insight. 2017;2(6):112. Doi: $10.1172 /$ jci.insight. 92002

19. Anoop SV Shah, David AMc Allister, Anne Cruikshank, Mary Stoddart Paul O Collinson, Alasdair J Gray, et al., High sensitivity cardiac troponin and the under-diagnosis of myocardial infarction in women: prospective cohort study. BMJ.2015;350:1-8. Doi: org/10.1136/bmj. g7873

20. Mueller C, Giannitsis E, Christ M, Ordonez-Llanos J, deFilippi C, McCord J. Multicenter Evaluation of a 0-Hour/1-Hour Algorithm in the Diagnosis of Myocardial Infarction With High-Sensitivity Cardiac Troponin T. Ann Emerg Med. 2016;68(1):76-87. Doi: 10.1016/j. annemergmed.2015.11.013 
21. Furuhashi M, Saitoh S, Shimamoto K, Miura T. Fatty Acid-Binding Protein 4 (FABP4): Pathophysiological Insights and Potent Clinical Biomarker of Metabolic and Cardiovascular Diseases. Clin Med Insights Cardiol. 2015;8(3): 23-33. Doi: 10.4137/CMC.S17067

22. Liu HS, Xiao HS. MicroRNAs as potential biomarkers for gastric cancer World J Gastroenterol. 2014;20(34): 12007-12017. Doi: 10.3748/wjg. v20.i34.12007

23. Cha T, Cho S, Kim YT, Lee JH. Rapid aptasensor capable of simply diagnosing prostate cancer. Biosens Bioelectron. 2014;62:31-37. Doi: 10.1016/j.bios.2014.06.015

24. Jolly P, Formisano N, Tkac J, Kasak P, Frost C, Estrela P. LabelFree Impedimetric Aptasensor with Antifouling Surface Chemistry: a Prostate Specific Antigen Case Study. Sensors and Actuators B: Chemical. 2015;209:306-312. Doi: 10.1016/j.snb.2014.11.083

25.Stephan C, Ralla B, Jung K . Prostate-specific antigen and other serum and urine markers in prostate cancer. Biochimica et Biophysica Acta. 2014;1846(1):99-112. Doi: 10.1016/j.bbcan.2014.04.001

26.Zu K , Mucci L, Rosner BA, Clinton SK, Loda M , Stampfer MJ. A Prospective Study in the Prostate-Specific Antigen Era. J Natl Cancer Inst. 2014;106(2):1-10. Doi: 10.1093/jnci/djt430

27.http://drugline.org/img/term/prostate-specific-antigen-12208_0.jpg

28. Aggidis AG, Newman JD, Aggidis GA. Investigating pipeline and state of the art blood glucose biosensors to formulate next steps. Biosensors and Bioelectronics. 2015;74:243-262. Doi: 10.1016/j. bios.2015.05.071

29. Pavla Martinkova and Mirlosav Pohanka. Biosensors for Blood Glucose and Diabetes Diagnosis: Evolution, Construction, and Current Status. Biosensors. 2015;48(16):2509-2532. Doi: 10.1080/00032719.2015.1043661

30. Luca Cevenini, Maria Maddalena Calabrettaet, Elisa Michelini, Donato Calabria, Patrizia Simoni, Aldo Roda. Integrating Biochemiluminescence Detection on Smartphones: Mobile Chemistry Platform for Point-of-Need Analysis Anal. Chem. 2014;86(15):72997304. Doi: $10.1021 /$ ac502137s

31.Ji Won Lim, Dogyeong Ha, Jongwan Lee, Sung Kuk Lee, Taesung Kim Review of micro/nanotechnologies for microbial biosensors. Front Bioeng Biotechnol. 2015;3(61):1-13. Doi: 10.3389/fbioe.2015.00061
32. Ma X, Jiang Y, Jia F, Yu Y, Chen J, Wang Z. An aptamer-based electrochemical biosensor for the detection of Salmonella. J Microbiol Methods. 2014;98:94-98. Doi: 10.1016/j.mimet.2014.01.003

33. Kim G, Moon JH , Moh CY, Lim JG . A microfluidic nano-biosensor for the detection of pathogenic Salmonella. Biosensors and Bioelectronics. 2014;67:243-247. Doi: 10.1016/j.bios.2014.08.023

34. Mythili V. Embedded based food quality detection with biosensor technology. International Conference on Advanced Communication Control and Computing Technologies. 2016;333-336. Doi: 10.1109/ ICACCCT.2016.7831657

35. Tawil N, Sacher E, Mandeville R, Meunier M. Bacteriophages: biosensing tools for multi-drug resistant pathogens. Analyst. 2014;139(6):12241236. Doi: 10.1039/c3an01989f

36. Palmiro Poltronieri, Valeria Mezzolla, Elisabetta Primiceri, Giuseppe Maruccio. Biosensors for the Detection of Food Pathogens. Foods. 2014;3(3):511-526. Doi: 10.3390/foods3030511

37. Peng Zheng, Ming Li, Richard Jurevic, Scott K. Cushing, Yuxin Liu, Nianqiang Wu. A Gold Nanohole Array Based Surface Enhanced Raman Scattering Biosensor for Detection of Silver (I) and Mercury (II) in Human Saliva. Nanoscale. 2015;25:11005-11012. Doi:10.1039/ C5NR02142A

38. Onopiuk A, Tokarzewicz A, Gorodkiewicz E. Cystatin C: A Kidney Function Biomarker. Advances in Clinical Chemistry. 2015;68:57-69. Doi: 10.1016/bs.acc.2014.11.007

39. Lucian Rotariu, Florence Lagarde, Nicole Jaffrezic-Renault, Camelia Bala. Electrochemical biosensors for fast detection of food contaminants - trends and perspective. Trends in Analytical Chemistry. 2016;79:80-87. Doi: 10.1016/j.trac.2015.12.017

40. Bugatti S, Vitolo B, Caporali R, Montecucco C, Manzo A. B Cells in Rheumatoid Arthritis: From Pathogenic Players to Disease Biomarkers. Biomed Res Int. 2014;1-15. Doi: 10.1155/2014/681678

41.X Niu and G Chen. Clinical Biomarkers and Pathogenic-Related Cytokines in Rheumatoid Arthritis. Journal of Immunology Research. 2014:1-7. Doi: $10.1155 / 2014 / 698192$

42. Hamidreza Shirzadfar, Mustapha Nadi, Djilali Kourtiche, Yamada S, Thomas Hauet. Needle-type GMR sensor to estimate the magnetic properties of diluted ferrofluid for biomedicine application. IRBM. 2015;36(3):178-184. Doi: 10.1016/j.irbm.2015.01.014 